\section{Câncer de colo de útero em Alagoas: um estudo retrospectivo}

Cervical cancer in Alagoas: a retrospective study

Cáncer de cuello uterino en Alagoas: estudio descriptivo retrospectivo https://doi.org/10.28998/rpss.e02106010

Recebido em: 19/12/2020

Aceito em: 10/06/2021

Disponível online: 18/06/2021

Autor Correspondente:

Mayra Alencar da Silva

mayraalencars@outlook.com

Mayra Alencar da Silva ${ }^{1}$, Bruna Stefany Rebouças França ${ }^{2}$, João Paulo Oliveira de Almeida ${ }^{2}$, Thais Miranda Rodrigues ${ }^{2}$, Vanessa Silva Santos ${ }^{2}$, Amanda Karine Barros Ferreira Rodrigues ${ }^{2}$

${ }^{1}$ Centro Universitário Tiradentes. Maceió (AL), Brasil.

2 Universidade Federal de Alagoas/UFAL. Maceió (AL), Brasil.

\title{
Resumo
}

Objetivo: o câncer de colo do útero está diretamente relacionado com a infecção pelo Papilomavírus Humano e possui estratégias de prevenção como o exame Papanicolau e a vacinação contra o vírus. Analisar a cobertura das políticas de rastreamento e seus impactos na mortalidade do câncer de colo de útero em Alagoas. Método: foi feito um estudo retrospectivo, em outubro de 2020 , com os dados relativos à realização de exame citopatológico, colposcopia e biópsia de colo de útero dos municípios alagoanos entre 2013 e 2019 e mortalidade por câncer de colo de útero em Alagoas e no Brasil entre 2013 e 2018 extraídos do Departamento de Informática do Sistema Único de Saúde (DATASUS) e do Instituto Nacional de Câncer (INCA). Resultados: os dados evidenciam que o rastreamento pelo exame citopatológico vem demonstrando uma cobertura abaixo de $10 \%$ nos últimos anos quando comparado à população feminina alagoana preconizada para a realização. Além disso, as mortes por câncer de colo do útero estão estabilizadas, o que demonstra a ineficácia das políticas públicas. Conclusão: a mortalidade por câncer de colo de útero em Alagoas segue aumentando, sendo $120 \mathrm{em} \mathrm{2018,} \mathrm{o} \mathrm{que} \mathrm{evidencia} \mathrm{que} \mathrm{o} \mathrm{rastreamento} \mathrm{atual} \mathrm{não} \mathrm{cumpre} \mathrm{o}$ papel de diminuir a mortalidade, pois tem se mostrado insuficiente. Torna-se essencial o desenvolvimento e 0 fortalecimento de ações integradas, em todos os níveis de atenção, a fim de organizar o processo de rastreamento e ampliar a oferta de procedimentos da linha de cuidado de câncer de colo de útero.

Descritores: Neoplasias do Colo do Útero; Infecções por Papilomavírus; Saúde da mulher.

\section{Abstract}

Objective: cervical cancer is directly related to infection with the Human Papillomavirus and has prevention strategies such as the Pap smear and vaccination against the virus. Analyze the coverage of screening policies and their impact on cervical cancer mortality in Alagoas. Methods: a retrospective study was conducted in October 2020, with data on the performance of cytopathological examination, colposcopy and cervical biopsy in the municipalities of Alagoas between 2013 and 2019 and cervical cancer mortality in Alagoas and Brazil between 2013 and 2018 extracted from the Department of Informatics of the Unified Health System (DATASUS) and the National Cancer Institute (INCA). Results: the data shows that screening by cytopathological exam has shown coverage below $10 \%$ in recent years when compared to the female population of Alagoas recommended to be performed. Moreover, deaths from cervical cancer are stable, which demonstrates the ineffectiveness of public policies. Conclusion: mortality from cervical cancer in Alagoas continues to increase, with 120 in 2018, which shows that the current screening does not fulfill the role of reducing mortality, because it has proven to be insufficient. It is essential to develop and strengthen integrated actions, at all levels of care, in order to organize the screening process and expand the supply of cervical cancer care line procedures.

Descriptors: Papanicolaou Test; Uterine Cervical Neoplasms; Cancer.

\section{Resumen}

Objetivo: analizar la cobertura de las políticas de rastreo y sus impactos en la mortalidad por cáncer de cuello uterino en Alagoas. Método: se realizó un estudio retrospectivo, en octubre de 2020, con datos relacionados con la realización de examen citopatológico, colposcopia y biopsia cervical de los municipios de Alagoas entre 2013 y 2019 y mortalidad por cáncer de cuello uterino en Alagoas y Brasil entre 2013 y 2018 extraídos del Departamento de Informática del Sistema Único de Salud (DATASUS) y del Instituto Nacional del Cáncer (INCA). Resultados: los datos muestran que el rastreo por examen citopatológico ha mostrado una cobertura inferior al $10 \%$ en los últimos años en comparación con la población femenina de Alagoas recomendada para el procedimiento. Además, se estabilizan las muertes por cáncer de cuello uterino, lo que demuestra la ineficacia de las políticas públicas. Conclusión: la mortalidad por cáncer de cuello uterino en Alagoas sigue aumentando hasta llegar a 120 en 2018, lo que demuestra que el rastreo actual no cumple el papel de reducir la mortalidad, ya que se ha demostrado que es 
insuficiente. Es fundamental desarrollar y fortalecer acciones integradas, en todos los niveles de atención, con el fin de organizar el proceso de rastreo y ampliar la oferta de procedimientos de atención del cáncer de cuello uterino.

Descriptores: Neoplasias de Cuello Uterino; Infecciones por Papilomavirus; Salud de la mujer.

\section{Introdução}

A infecção pelo papilomavírus humano (HPV) está inter-relacionada com a fisiopatologia do câncer do colo do útero. ${ }^{1} \mathrm{O}$ desenvolvimento dessa neoplasia maligna, principalmente na zona de transição do colo do útero, depende da regulação negativa do controle do ciclo celular, do acúmulo de danos genéticos por oncoproteínas virais e da ineficácia imunológica frente à infecção persistente pelo HPV. ${ }^{2}$

O HPV é um vírus DNA que se apresenta em genótipos de baixo e alto riscos. Os tipos 6 e 11 são os mais prevalentes de baixo risco, não sendo capazes de causar câncer, mas sim outros tipos de lesões e infecções. ${ }^{1,3}$ Dentre os de alto risco $(16,18,31,33,35$, $39,45,51,52,56,58,59,68,73$ e 82), capazes de infectar as membranas cutâneas e as mucosas em diferentes sítios anatômicos, ${ }^{3-4}$ os tipos 16 e 18 são mais prevalentes no desenvolvimento de câncer do colo do útero, possuem proteínas, E6 e E7, que inibem os genes supressores tumorais p53 e RB, respectivamente, e induzem o processo carcinogênico. O tipo 16, em específico, causa o carcinoma de células escamosas e o 18 causa o adenocarcinoma, que é mais agressivo, apesar de menos prevalente. ${ }^{5}$

O câncer do colo do útero é o quarto câncer mais comum em mulheres em todo o mundo e a principal causa de morte por câncer em alguns dos países mais pobres. ${ }^{6}$ Cerca de $90 \%$ das mortes são registradas em países de média e baixa rendas devido a diagnósticos tardios e ao não recebimento ou conclusão dos regimes de tratamento prescritos. Por outro lado, os países desenvolvidos possuem programas de rastreamento, instalações de diagnóstico e tratamento eficientes e acessíveis, assim apresentando morbidade e mortalidade menores. $^{7}$ No Brasil, a epidemiologia do câncer de colo de útero é impactada por suas proporções continentais e importante diversidade socioeconômica, resultando em Estados com mortalidade por câncer de colo de útero em ascensão, como Alagoas. ${ }^{3}$

\section{Existem duas estratégias de prevenção} comprovadamente eficazes nesse tipo de câncer que são a vacinação contra o HPV, preferencialmente em meninas dos nove aos 14 anos e meninos dos 11 aos 14 anos, e o rastreamento cervical com teste primário de HPV em mulheres dos 25 aos 64 anos por colpocitologia oncótica, seguido do tratamento de lesões précancerosas, caso detectadas. ${ }^{5,8}$

Além das medidas de acompanhamento, determinadas pela estratificação de Neoplasias Intraepiteliais Cervicais (NIC) em baixo (I), moderado (II) e alto grau (III) de displasia, há um protocolo de condutas específicas a serem seguidas de acordo com o grau de NIC estabelecido no exame de colpocitologia oncótica. Caso o exame declare NIC I, significa que a mulher repetirá o exame seis meses depois; no caso de NIC II ou III, é direcionada imediatamente para a colposcopia seguida de biópsia, podendo ser por conização e posteriores tratamentos caso a malignidade da neoplasia seja confirmada. ${ }^{8}$

O câncer do colo do útero é evitável e curável. Apresenta 92\% de taxa de sobrevivência de cinco anos, se detectado em um estágio inicial, e $71 \%$, se analisados todos os estágios. ${ }^{4}$ Dessa forma, os desfechos de previsão de mortalidade e sobrevida têm sido associados a variáveis sociodemográficas como o resultado do baixo nível socioeconômico e da cobertura inadequada do rastreamento do câncer do colo do útero no Brasil. ${ }^{7}$

Ao revelar o grande problema de saúde pública que são a carência de educação sexual, a fragilidade das políticas de cunho informativo a respeito dos exames preventivos e a não totalidade da cobertura pelo Sistema Único de Saúde (SUS), ${ }^{9}$ tornou-se o objetivo deste trabalho analisar a cobertura do rastreamento, bem como a sua relação com as taxas de mortalidade por câncer de colo de útero de Alagoas. 


\section{Método}

Trata-se de um estudo de coorte do tipo retrospectivo, realizado em outubro de 2020, com dados secundários, assim dispensando a apreciação de um Comitê de Ética em Pesquisa (CEP).

Para identificar o rastreio dos casos de câncer de colo de útero em Alagoas, foi utilizado o Departamento de Informática do Sistema Único de Saúde (DATASUS), por meio do Tabulador de Dados para Ambiente Internet (TABNET), com informações disponibilizadas pelo Sistema de Informações Ambulatoriais do Sistema Único de Saúde (SIA/SUS), presente no setor de assistência à saúde. Foram extraídos a quantidade, enquanto variável dependente, e os locais de atendimento dos exames citopatológicos, colposcopias e biópsias de colo de útero, como variável independente, no período de 2013 a 2019, dos municípios de Alagoas.

A fim de identificar a mortalidade $e$ as características do câncer de colo de útero em Alagoas, foram selecionadas, no tabulador do Atlas de Mortalidade por Câncer do Instituto Nacional de Câncer (INCA), como variáveis dependentes, as taxas de mortalidade no período de 2013 a 2018, no gênero feminino, no Brasil e em Alagoas, considerando, como variável independente, o CID 10 referente ao colo do útero (C53 - Neoplasia maligna do colo do útero; C53.0 Neoplasia maligna do endocérvix; C53.1 - Neoplasia

maligna do exocérvix; C53.8 - Neoplasia maligna do colo do útero com lesão invasiva; C53.9 - Neoplasia maligna do colo do útero, não especificado). Utilizou-se o desviopadrão, uma medida de dispersão, a fim de verificar a uniformidade dos dados obtidos. Para a análise, os dados coletados foram tabulados em planilhas eletrônicas, utilizando, como variáveis, os tipos de procedimentos realizados por ano e analisados a partir da literatura.

\section{Resultados}

O rastreamento do câncer de colo de útero ocorre de diferentes formas, sendo formado por um fluxo do exame citopatológico do colo de útero, da colposcopia e da biópsia de colo uterino. Na figura 1, está presente a cobertura desses procedimentos de rastreamento em Alagoas entre 2013 e 2019. A adesão à realização de exame citopatológico está apresentando uma queda nos últimos anos, especialmente em $2018(n=25.661)$ e 2019 ( $n=25.756)$, o que equivale a $3,26 \%$, em 2018, e a $3,27 \%$, em 2019, das mulheres alagoanas entre 24 e 64 anos $(n=785.379)$ de acordo com o censo demográfico do IBGE de 2010. Como há um fluxo de cuidado, as taxas baixas de exame citopatológico são refletidas, diretamente, em taxas baixas de colposcopia, em 2018 ( $n=10.388)$ e 2019 ( $n=12.560)$ e biópsia do colo uterino, em $2018(n=1.466)$ e 2019 ( $n=1752)$.

Figura 1- Procedimentos para o rastreamento de câncer de colo de útero em Alagoas por ano.

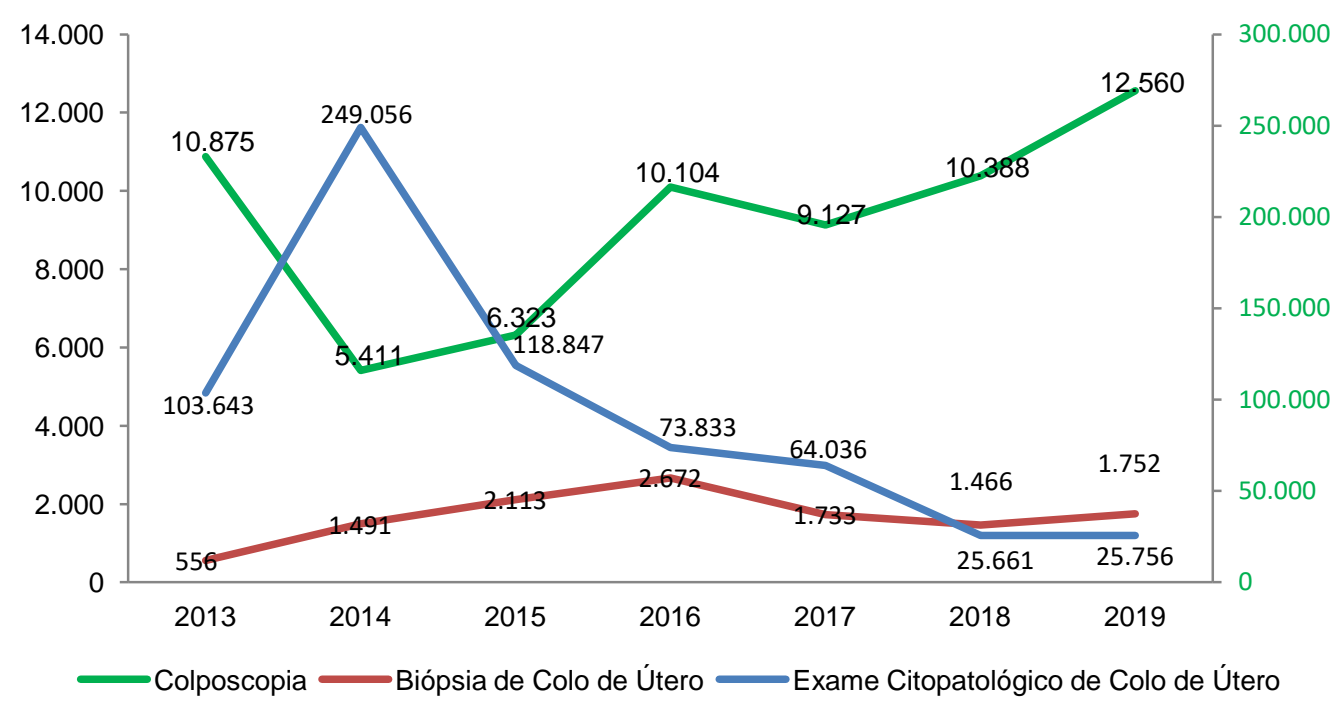

Fonte: Sistema de Informações Ambulatoriais do Sistema Único de Saúde (SIA/SUS). 
A quantidade de óbitos absolutos por câncer de colo de útero em Alagoas (Tabela 1) segue crescente, embora não tenha apresentado variações elevadas no período entre 2013 e 2018, o que é evidenciado pelo baixo desvio-padrão $(\sigma=12,11)$ quando há a análise desses valores. Quando comparado ao número absoluto do Brasil, o perfil assemelha-se visto que não há altas variações no período entre 2013 e 2018 com desviopadrão baixo $(\sigma=465,79)$.

Tabela 1- Número absoluto de óbitos por câncer de colo do útero.

\begin{tabular}{ccc}
\hline Ano & Brasil & Alagoas \\
\hline 2013 & 5430 & 85 \\
2014 & 5448 & 101 \\
2015 & 5727 & 103 \\
2016 & 5847 & 97 \\
2017 & 6385 & 112 \\
2018 & 6526 & 120 \\
\hline
\end{tabular}

Fonte: Atlas de Mortalidade por Câncer do Instituto Nacional de Câncer (INCA).

Figura 2- Taxas de mortalidade/100 mil por câncer de colo de útero no Brasil e em Alagoas entre 2013 e 2018.

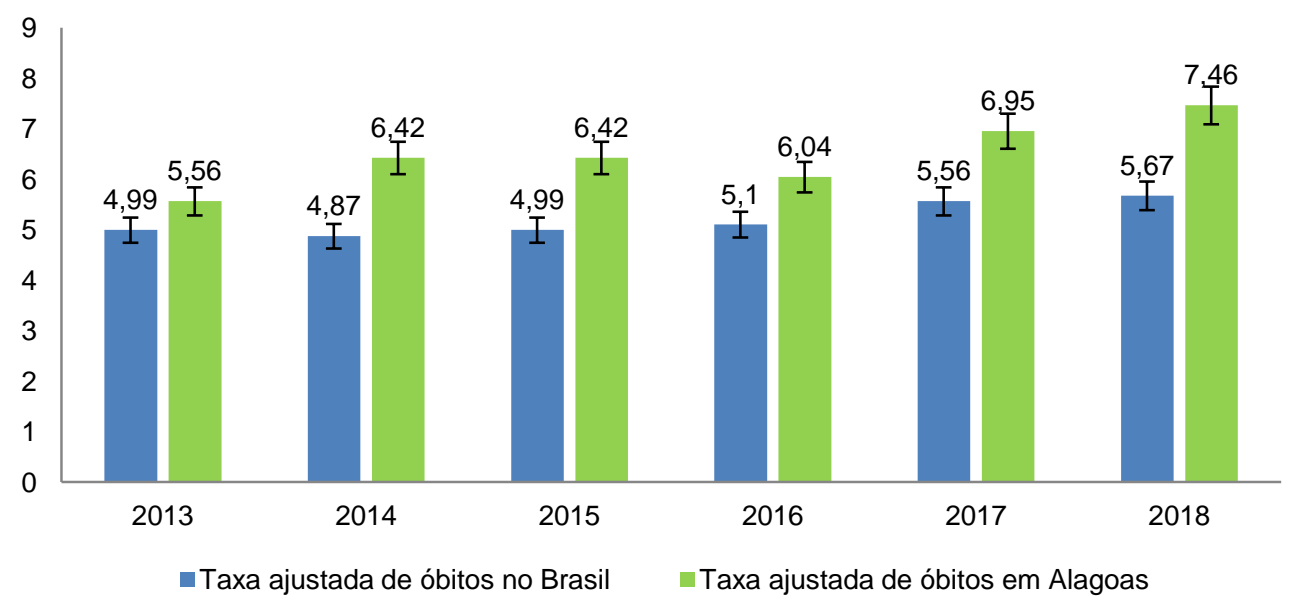

Fontes: MS/SVS/DASIS/CGIAE/Sistema de Informação sobre Mortalidade(SIM), MP/Fundação Instituto Brasileiro de Geografia e Estatística - IBGE, MS/INCA/Conprev/Divisão de Vigilância.

\section{Discussão}

Neste estudo, pôde-se verificar que Alagoas apresenta um quadro de subnotificação de casos de câncer de colo de útero, haja vista uma decrescente taxa de realização de exames de rastreamento, principalmente em 2018 e 2019, e uma crescente taxa de mortes por câncer de colo de útero.

Apesar do rastreamento do câncer de colo de útero ser responsável por diminuir as taxas de óbitos e devido ao tratamento precoce promover melhores prognósticos, nota-se que esse controle, ainda, é um
Ao comparar as taxas de mortalidade ajustadas, de acordo com o perfil epidemiológico, em Alagoas e no Brasil, nota-se que Alagoas está acima da taxa de mortalidade do Brasil, principalmente em 2018, em que Alagoas apresenta uma alta taxa de mortalidade ajustada $(n=7,46)$ quando comparada à do Brasil $(n=$ $5,67)$ (Figura 2). 
recomendados para essa prática, além do conhecimento sobre como orientar e encaminhar para o tratamento, nos níveis de atenção secundária e terciária à saúde, as mulheres de acordo com os resultados dos exames e garantir o seu seguimento. ${ }^{8}$

O volume de exames de rastreamento concluídos em Alagoas é baixo e está em queda, tendência comum em Estados pobres e com mulheres mais carentes. ${ }^{11}$ Isso principalmente ocorre em virtude da falta de orientação por parte dos Serviços de Saúde sobre o que é o câncer de colo de útero, a importância do exame preventivo para mulheres e sua realização com periodicidade na população de risco, além da carência de educação sexual e a dificuldade em estabelecer uma cobertura completa pelo SUS. ${ }^{12}$ Tal achado é notável nos valores baixos na realização de procedimentos de rastreamento em Alagoas, destacando-se a queda nos anos de $2018(n=23.661)$ e $2019(n=25.756)$, quando comparados aos anos de $2016(n=73.833)$ e 2017 $(n=64.036)$ no que se refere à coleta para exames citopatológicos do colo de útero.

A queda no rastreamento é preocupante, uma vez que 0 rastreamento, quando direcionado a uma população assintomática, detecta as lesões sugestivas ou precursoras de câncer e está apresentando uma baixa cobertura das mulheres sexualmente ativas no Estado de Alagoas, considerando que, de acordo com o Censo Demográfico de 2010, há 785.379 mulheres na faixa etária entre 24 e 64 anos, estabelecida pelo Ministério da Saúde para a realização do exame citopatológico e, desde do ano de 2016, a cobertura não alcança $10 \%$ do grupo referido, o que se torna preocupante. $^{13}$

As recomendações para o rastreamento seguem a lógica de quando os dois exames citopatológicos subsequentes com intervalo de seis (para as mulheres com 30 anos ou mais) ou 12 meses (para as mulheres com menos de 30 anos) forem negativos, a mulher deverá retornar à rotina de rastreamento trienal. No entanto, caso o resultado de alguma citologia for sugestivo de lesão intraepitelial ou câncer, a mulher deverá ser referenciada à unidade de referência para a colposcopia. No caso de a colposcopia mostrar achados anormais (sugestivos de NIC II/III) ou suspeitos de invasão, deve-se realizar a biópsia que, por sua vez, se apresentar como resultado NIC II/III ou câncer, a conduta será específica para esse resultado. ${ }^{11}$

Os resultados deste estudo mostram que as colposcopias e as biópsias de colo uterino estão sendo realizadas em uma escala pequena quando comparadas ao exame citopatológico. Assim, revelam uma disparidade entre a confirmação ou a exclusão do diagnóstico de câncer de colo de útero, evidenciando uma falha no processo de referenciamento da APS, tendo em vista que o fluxo de encaminhamento e a continuidade da atenção são essenciais para a garantia da integralidade do cuidado, visando ao controle do câncer do colo do útero. Esse controle depende do planejamento, da coordenação e da execução dos gestores locais do SUS e de suas equipes técnicas, o que confirma a fragilidade nas ações de saúde da mulher em Alagoas, principalmente no que tange às demandas para as prevenções primárias e secundárias. $^{14}$

Estudos semelhantes relataram as dificuldades no seguimento quando identificadas as lesões sugestivas no colo do útero durante o exame citopatológico para a investigação diagnóstica e 0 tratamento, caso necessário. Além disso, destacaram a necessidade de direcionar a faixa etária e a periodicidade para a realização dos exames, visando a atingir o públicoalvo. $^{15-16}$

O início precoce de tratamento pode contribuir para melhores resultados na terapêutica. Quando tardio muitas vezes, após 60 dias, prazo estabelecido pelo SUS -, afeta diretamente a sobrevida das mulheres por limitar as perspectivas de tratamentos. Diante disso, as taxas de mortalidade por câncer do colo do útero em Alagoas e no Brasil continuam expressivas. A partir disso, é notável que os programas de rastreamento precoce não estão conseguindo ampliar a sua cobertura, além da morosidade associada à falta da continuidade no processo de cuidado, que afeta diretamente as taxas de mortalidade. ${ }^{15-16}$

A estabilidade nos valores absolutos de câncer de colo de útero, tanto em Alagoas quanto no Brasil, pode ser explicada pelo fato de esse tipo de câncer ser comum em países de baixa e média rendas, 
considerando que não são apresentadas variações altas durante o período analisado. Como mostra este estudo, torna-se evidente uma baixa eficácia na aplicação de políticas públicas de rastreamento de câncer de colo de útero, realizada por meio dos exames preconizados pelo Ministério de Saúde, para mulheres com vida sexual ativa. $^{17}$

Outros estudos avaliaram essa mesma variação e reportaram existir uma queda nos períodos de 19802010 e 2003-2012. A justificativa é que essas variações são resultados de programas de ações afirmativas do governo iniciados na década de 1940 para combater essa patologia. ${ }^{17}$

$\mathrm{Na}$ análise das taxas de mortalidade de câncer de colo de útero em Alagoas e no Brasil, nota-se que o Estado de Alagoas está acima da taxa nacional em todo o período analisado, o que pode ser explicado pelo Estado apresentar o menor Índice de Desenvolvimento Humano (IDH) do Brasil (IDH = 0,631) e possuir cerca de $96,10 \%$ dos municípios em alta ou muito alta vulnerabilidade social. ${ }^{13,18}$

A dificuldade no rastreamento torna-se um risco porque o câncer de colo de útero é o terceiro tipo de câncer mais comum em mulheres, atrás apenas do câncer de mama e do câncer colorretal, caso não se considere o câncer de pele não melanoma. E o risco aumenta quando se considera que ele também é a quarta causa mais frequente de morte por neoplasia entre as mulheres no Brasil e no mundo. ${ }^{8,19}$ Como as principais dificuldades relacionadas ao diagnóstico e ao início de tratamento do câncer de colo uterino são o acesso ao Papanicolau na Atenção Básica (AB), a percepção por parte das usuárias que utilizam os serviços no SUS é de que estes possuem baixa resolutividade, o que faz a procura por atendimento ser reduzida e o tratamento, fragmentado, comprometendo a integralidade do cuidado.

\section{Conclusão}

A análise do rastreamento e da mortalidade do câncer de colo uterino em Alagoas revelou que está ocorrendo um aumento das taxas de mortalidade, mesmo diante da subnotificação de casos, o que denuncia uma falha no processo de rastreamento e de referenciamento da APS.

Dito isso, torna-se de fundamental importância o fortalecimento de ações integradas, por meio de Rede de Atenção à Saúde, entre os níveis de complexidade de atenção, atenção primária, secundária e terciária, garantindo a promoção, a prevenção e a recuperação para as mulheres de Alagoas, ampliando o acesso à informação e à comunicação, por meio de folhetos e spots, além de reduzir as dificuldades de acesso aos serviços de saúde a fim de organizar o processo de rastreamento e ampliar a oferta de procedimentos da linha de cuidado de câncer de colo de útero com o apoio de equipes humanizadas que atendam 0 paciente integralmente - tal como o princípio organizacional do SUS.

Por meio de tais políticas, haverá uma melhora na articulação dos serviços em seus diferentes níveis de complexidade, permitindo um aumento substancial no diagnóstico de casos e uma ampliação no acesso às possíveis formas de tratamento. Campanhas de publicidade devem ser realizadas visando à percepção das usuárias do SUS sobre os serviços ofertados, garantindo uma maior procura por atendimento e adesão ao tratamento. Este estudo apresenta limitações como o pequeno número da amostra dos resultados e o acesso à informação e ao aprofundamento nos casos individuais, além do recorte temporal analisado. Dessa forma, novos estudos devem ser realizados com o intuito de esclarecer as vulnerabilidades em saúde e no processo assistencial à saúde, haja vista as limitações deste estudo, para a elaboração de estratégias e políticas de saúde voltadas para o rastreamento e a prevenção desse câncer.

\section{Referências}

1. Okamoto $C T$, Faria $A A B$, Sater AC, Dissenha BV, Stasievski BS. Profile of knowledge on hpv and its prevention among students at a private University in Curitiba. Rev Bras Educ Med. 2016 Oct/Dec; 40(4):611-20. Doi: 10.1590/1981$52712015 \mathrm{v} 40 \mathrm{n} 4 \mathrm{e} 00532015$

2. Wang R, Pan W, Jin L, Huang W, Li Y, Wu D, et al., Human papillomavirus vaccine against cervical cancer: Opportunity and challenge. Cancer Letters. 2020 Feb; 471:88-102. Doi: 10.1016/j.canlet.2019.11.039 
3. Colpani V, Falcetta FS, Bidinotto AB, Kops NL Falavigna M, Hammes LS, et al. Prevalence of human papillomavirus (HPV) in Brazil: a systematic review and meta-analysis. PLoS One. 2020 Feb; 15(2):e0229154. Doi: 10.1371/journal.pone.0229154

4. Sharma A, Kulkarni V, Bhaskaran U, Singha M, Mujtahedi S, Chatrath A, et al. Profile of cervical cancer patients attending Tertiary Care Hospitals of Mangalore, Karnataka: A 4 year retrospective study. J Nat Sci Biol Med. 2017 Jan/June; 8(1):125-9. Doi: 10.4103/0976-9668.198354

5. Federação Brasileira das Associações de Ginecologia e Obstetrícia. HPV [Internet]. Rio de Janeiro: FEBRASGO; 2017 [cited 2020 Aug 10]. Available from:

https://www.febrasgo.org.br/pt/noticias/item/120$\underline{\mathrm{hpV}}$

6. Canfell K. Towards the global elimination of cervical cancer. Papillomavirus Res. 2019 Dec; 8:100170. Doi: $10.1016 / j . p v r .2019 .100170$

7. Dantas DB, Costa TL, Silva ASA, Gomes FC, Melo-Neto JS. Mortality from cervical cancer in Brazil: an ecological epidemiologic study of a 22-year analysis. Ecancer. 2020; 14:1064. Doi: 10.3332/ecancer.2020.1064

8. Ministério da Saúde (BR), Instituto Nacional de Câncer José Alencar Gomes da Silva. Diretrizes Brasileiras para o rastreamento do câncer do colo do útero [Internet]. Rio de Janeiro: INCA; 2016 [cited 2020 Aug 10]. Available from: http://bvsms.saude.gov.br/bvs/publicacoes/inca/rastr eamento cancer colo utero.pdf

9. Cruz NMRA, Cruz KEA, Silva CAL. Uterus cervix cancer mortality in the state of Bahia, brazil, between 1996 and 2012. Rev Baiana Saúde Pública. 2018 Oct/Dec; 42:624-39. Doi: 10.22278/23182660.2018.v42.n4.a2807

10. Ribeiro CM, Dias MBK, Pla MAS, Correa FM, Russomano FB, Tomazelli JG. Parameters for programming line of care procedures for cervical cancer in Brazil. Cad Saúde Pública. 2019 July; 35(6):e00183118. Doi: 10.1590/0102$311 \times 00183118$

11. Peroni FMA, Lindelow $M$, Souza DO, Sjoblom $M$. Realizing the right to health in Brazil's Unified Health System through the lens of breast and cervical cancer. Int J Equity Heal. 2019; 18:39. Doi: $10.1186 / \mathrm{s} 12939-019-0938-x$

12. Silva LR, Almeida CAPL, Sá GGM, Moura LKB, Araújo, ETH. Health education as a strategy to prevent cervical cancer: review integrative. Rev Pre Infec e Saúde [Internet]. 2017 [cited 2020 Aug 10]; 3(4):35-45. Available from: http://www.ojs.ufpi.br/index.php/nupcis/article/view/ $\underline{6708}$

13. Ministério do Planejamento, Orçamento e Gestão (BR), Instituto Brasileiro de Geografia e Estatística. Censo Demográfico 2010. Distribuição da população por sexo, segundo os grupos de idade [Internet]. Rio de Janeiro: IBGE; 2011 [cited 2020 Aug 10]. Available from: https://censo2010.ibge.gov.br/sinopse/webservice/fr m piramide.php?codigo $=27$
14. Pinafo $E$, Nunes EFPA, Carvalho BG, Mendonça FF, Domingos CM, Silva CR. Management problems and strategies: the vulnerability of small-sized municipalities. Ciênc Saúde Colet. 2020 May; 25(5):1619-28. Doi: 10.1590/141381232020255.34332019

15. Corrêa CSL, Lima AS, Leite ICG, Pereira LC, Nogueira $M C$, Duarte DAP, et al. Cervical cancer tracking in Minas Gerais: assessment of data from Cervical Cancer Information System (SISCOLO). Cad Saúde Colet. 2017 July/Sept; 25(3):315-23. Doi: $10.1590 / 1414-462 \times 201700030201$

16. Maia MN, Silva RPO, Santos LPR. The cervix cancer tracking organization by a Family Health team in Rio de Janeiro, Brazil. Rev Bras Med Fam Comunidade. 2018 Jan/Dec;13(40):1-10. Doi: $10.5712 /$ rbmfc13(40) 1633

17. Carvalho PG, O'Dwer G, Rodrigues NCP. Health assistance path of women between diagnosis and treatment initiation for cervix cancer. Saúde em Debate. 2018 July/Sept; 42(118):687-701. Doi: 10.1590/0103-1104201811812

18. Ministério da Economia (BR), Instituto de Pesquisa Econômica Aplicada. Atlas da vulnerabilidade social nos municípios brasileiros [Internet]. Brasília: IPEA; 2015 [cited 2020 Aug 10]. Available from: http://ivs.ipea.gov.br/index.php/pt/sobre

19. Kashyap N, Krishnan N, Kaur S, Ghai S. Risk factors of cervical cancer: a case-control study. Asia Pac J Oncol Nurs. 2019 July/Sept; 6(3):308-14. Doi: 10.4103/apjon.apjon_73_18.

\section{Citação:}

Silva MA et al. Câncer de colo de útero em Alagoas: um estudo retrospectivo. Rev. Port. Saúde e Sociedade. 2021;6(único):e02106010. Doi:10.28998/rpss.e02106010. 